Ivi Vasconcelos Elias

\title{
O MITO DE SÍSIFO: \\ a mediação do processo de paz na Irlanda do Norte e a assinatura do Acordo de Sexta Feira Santa
}

\section{Dissertação de Mestrado}

Dissertação apresentada como requisito parcial para a obtenção do grau de Mestre pelo Programa de Pós-Graduação em Relações Internacionais do Instituto de Relações Internacionais da PUC-Rio.

Orientador: Prof. Dr. Nizar Messari

Rio de Janeiro

Agosto de 2009 


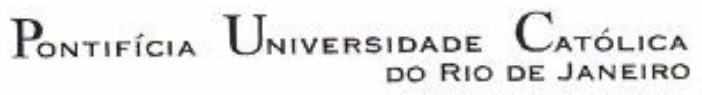

Ivi Vasconcelos Elias

O MITO DE SÍSIFO:

a mediação do processo de paz na Irlanda do Norte e a assinatura do Acordo de Sexta Feira Santa

\begin{abstract}
Dissertação apresentada como requisito parcial para a obtenção do grau de Mestre pelo Programa de PósGraduação em Relações Internacionais da PUC-Rio. Aprovada pela Comissão Examinadora abaixo assinada.
\end{abstract}

Prof. Nizar Messari

Orientador

Instituto de Relações Internacionais - PUC-Rio

Prof. - Miriam Gomes Saraiva Instituto de Filosofia e Ciências Humanas - UERJ

Prof. a Mônica Herz

Instituto de Relações Internacionais - PUC-Rio

Nizar Messari

Coordenador Setorial do Centro de Ciências Sociais - PUC-Rio

Rio de Janeiro, 14 de agosto de 2009 
Todos os direitos reservados. É proibida a reprodução total ou parcial do trabalho sem autorização da autora, do orientador e da universidade.

\section{Ivi Vasconcelos Elias}

Graduou-se em Relações Internacionais pela Universidade de Vila Velha (UVV) em 2003 e em Direito pela Universidade Federal do Espírito Santo (UFES) em 2008. Integra o corpo docente da graduação em Relações Internacionais da Universidade Candido Mendes (UCAM). Tem interesse pelas áreas de Segurança Internacional, Resolução de Conflitos, Teoria das Relações Internacionais e Ciência Política.

Ficha catalográfica

Elias, Ivi Vasconcelos

O mito de Sísifo: a mediação do processo de paz na Irlanda do Norte e a assinatura do Acordo de Sexta Feira Santa / Ivi Vasconcelos Elias; orientador: Nizar Messari. - Rio de Janeiro: PUC, Instituto de Relações Internacionais, 2006.

237f.: il. ; $30 \mathrm{~cm}$

Dissertação (mestrado) - Pontifícia Universidade Católica do Rio de Janeiro, Instituto de Relações Internacionais.

Inclui referências bibliográficas.

1. Relações internacionais - Teses. 2. Segurança Internacional. 3. Resolução de Conflitos. 4. Mediação Internacional. 5. Irlanda do Norte. 6. Acordo de Sexta Feira Santa. I. Messari, Nizar. II. Pontifícia Universidade Católica do Rio de Janeiro. Instituto de Relações Internacionais. III. Título. 


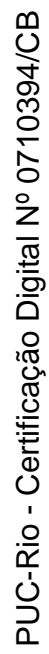

Para meu pai, Arlindo Elias Filho (in memorian), fonte permanente de amor e força. 


\section{Agradecimentos}

Ao meu orientador, Nizar Messari, pela paciência e generosidade que extrapolaram o papel de orientador e transformaram o acompanhamento desta dissertação em um ato de humanidade e amizade.

Às professoras Mônica Herz e Miriam Saraiva pela disponibilidade em participar da banca examinadora.

A minha mãe, Tania, e meus irmãos, Arlindo Neto e Gabriel, por serem a base que me possibilita traçar o meu próprio caminho.

Aos amigos que compartilharam o cotidiano do lar comigo e que se tornaram parte da minha família carioca: Marcela Vecchione, Erwin Xavier, Helena Massote, Kelly França, Daniel Aragão e Diana Aguiar. Em especial à Maíra Siman pela enorme generosidade e apoio imprescindível na reta final.

Aos amigos feitos na minha convivência acadêmica que se tornaram companheiros de vida: Cristina Alexandre, Marcelo Valença, Cristina Vinciprova, Tathiana Haddad e Renata Barbosa. Em especial à Mariana Carpes e Ana Carolina Delgado.

Aos colegas do IRI pelas trocas de idéias e convivência estimulante.

Aos funcionários do IRI pela presteza e atenção. Em especial a Luciana Varanda, a Natacha Oliveira e a saudosa Maria Helena Marques.

Aos meus amigos capixabas de sempre e para sempre, principalmente Poliano Bastos, Augusto Mozine, Camilla Magalhães e Diana Carvalhinho.

Aos meus alunos por terem se tornado um dos meus maiores incentivos para persistir na carreira acadêmica.

À CAPES, CNPq e PUC-Rio pelos auxílios concedidos sem os quais não seria possível a realização deste trabalho. 


\section{Resumo}

Elias, Ivi Vasconcelos. Messari, Nizar. O mito de Sísifo: a mediação do processo de paz na Irlanda do Norte e a assinatura do Acordo de Sexta Feira Santa. Rio de Janeiro, 2009. 237p. Dissertação de mestrado Instituto de Relações Internacionais, Pontifícia Universidade Católica do Rio de Janeiro.

A presente dissertação faz uso do instrumental teórico da Resolução de Conflitos a fim de analisar o gerenciamento do conflito que opõe os grupos nacionalista católico e unionista protestante na Irlanda do Norte. Tal processo culminou com a assinatura do Acordo de Sexta Feira Santa em 10 de abril de 1998. A estratégia aplicada nas negociações previa a atuação simultânea em duas frentes: na elaboração de um acordo que estabelecia o compartilhamento de instituições políticas e na construção da confiança entre as partes concentrada na questão da deposição de armas pelos grupos paramilitares. Essa dissertação busca problematizar a mediação como um processo de resolução de conflitos de natureza elitista que encara o conflito como um problema essencialmente de barganha. Compreende-se que a mediação não encoraja a reconciliação entre as comunidades antagônicas e a geração de uma cultura de cooperação política, falhando em promover a confiança entre as partes e ocasionando impasses na implementação do acordo de paz. Embora a mediação tenha sido fundamental para oferecer uma alternativa política para as partes beligerantes, o conflito na região permaneceu latente com a escalada da violência em momentos cruciais de progresso das negociações. Este estudo conclui que a concretização do acordo deveu-se muito ao esgotamento da sociedade civil e ao reconhecimento das partes da impossibilidade de vencer o conflito pela via armada. Essa conclusão aponta a necessidade de se complementar a abordagem elitista com iniciativas de resolução de conflitos constituídas no seio da sociedade civil para explicar a evolução do processo de paz na Irlanda do Norte rumo à transformação do conflito e à construção de uma paz positiva.

\section{Palavras-chave}

Segurança Internacional; Resolução de Conflitos; Mediação Internacional; Irlanda do Norte; Acordo de Sexta-Feira Santa. 


\section{Abstract}

Elias, Ivi Vasconcelos. Messari, Nizar (Advisor). The myth of Sisyphus: the mediation of the Northern Ireland peace process and the signature of the Good Friday Agreement. Rio de Janeiro, 2009. 237p. MSc. Dissertation - Instituto de Relações Internacionais, Pontifícia Universidade Católica do Rio de Janeiro.

This dissertation analyzes from a Conflict Resolution perspective the process of conflict management between catholic nationalists and protestant unionists in Northern Ireland. This process ended with the signature of the Good Friday Agreement in April of 1998. The strategy employed in the negotiations presupposed a twin track approach: the elaboration of a power sharing political arrangement and the implementation of confidence building measures for the decommissioning of arms by paramilitary groups. This dissertation aims to problematize mediation as an elitist conflict resolution process that considers conflict essentially in terms of a bargaining problem. Mediation is understood as a process that does not encourage the reconciliation of antagonist communities and the generation of a culture of cooperation, failing to promote confidence between the parties and imposing setbacks in the implementation of the peace agreement. Although mediation was able to offer to the belligerents parties a political alternative, the conflict in the region remained latent, with the escalation of violence during crucial moments when the negotiations were moving forward. This study concludes that the peace agreement was achieved much due to the disengagement of civil society in the conflict and the recognition of the parties that they would not win the conflict by forceful means. This conclusion points to the necessity of complementing the elitist approach with civil society conflict resolution initiatives in order to explain the evolution of the peace process in Northern Ireland towards conflict transformation and positive peace.

\section{Keywords}

International Security; Conflict Resolution; International Mediation; Northern Ireland; Good Friday Agreement 


\section{Sumário}

$\begin{array}{ll}1 . & 13\end{array}$

2. Resolução de Conflitos e Relações Internacionais: 19 quadro analítico para o estudo do processo de paz na Irlanda do Norte.

2.1 A Resolução de Conflitos e as Relações Internacionais 19

2.1.1 Gerenciamento de conflitos (conflict management) 23

2.1.2 Resolução de Conflitos (conflict resolution) 26

2.1.3 Transformação de Conflitos (conflict transformation) 29

2.2 O estudo do processo de paz na Irlanda do Norte 33

2.2.1 Abordagens de "cima para baixo" (top-down) 34

2.2.2 Abordagens de "baixo para cima" (bottom-up) 37

2.3 O modelo de mediação internacional de gerenciamento 44

2.3.1 A natureza da mediação 45

2.3.2 A mediação e o uso da força 48

2.3.3 Tipos de mediador 53

2.3.3.a Indivíduos 53

2.3.3.b Estados 55

2.3.3.c Organizações Internacionais, regionais e não- 57 governamentais

2.3.4 O aspecto comportamental na mediação 59

2.3.5 Avaliando a mediação internacional 61

3. $O$ conflito na Irlanda do Norte e a construção do 65 processo de paz

3.1 O conflito na Irlanda do Norte 66

$\begin{array}{lll}3.2 & \text { Os principais atores do conflito } & 76\end{array}$

$\begin{array}{lll}3.2 .1 & \text { Unionistas, lealistas, protestantes } & 77\end{array}$

$\begin{array}{lll}3.2 .2 & \text { Nacionalistas, republicanos, católicos } & 78\end{array}$

$\begin{array}{lll}3.2 .3 & \text { Reino Unido } & 79\end{array}$

3.2.4 República da Irlanda 82

3.2.5 O contexto internacional 82 
3.3 Construindo o processo de paz (1990-1996) 85

3.3.1 O Sinn Féin e o governo britânico 88

3.3.2 A articulação Hume-Adams 93

3.3.3 A Declaração de Downing Street 95

3.3.4 Os Framework Documents 104

3.3.5 A questão do desarmamento e o fim do cessar-fogo do 107 IRA

4. O processo de mediação do Acordo de Sexta Feira 119

Santa e seu impacto na construção da paz

4.1 O processo de mediação do GFA 120

4.1.1 A escolha e aceitação dos mediadores 120

4.1.2 A pré-negociação: a busca pelo estabelecimento de 123

$\begin{array}{lll}\text { 4.1.3 A definição da agenda preliminar para as negociações } & 128\end{array}$

4.1.4 Eleições e o novo impulso para o processo de paz 132

4.1.5 O início das negociações substantivas 139

4.1.6 Avaliando a mediação do GFA 154

4.2 O Acordo de Sexta Feira Santa (Good Friday Agreement 160 - GFA)

4.2.1 As disposições do Acordo 162

4.3 A continuidade do processo de paz: a implementação 165

4.3.1 As dificuldades na implementação 168

4.3.2 Avaliando o GFA 175

5. Conclusão 180

6. Referências Bibliográficas 188

7. Anexo 


\section{Lista de abreviaturas}

AIA - Anglo-Irish Agreement

APNI - Alliance Party of Northern Ireland

BIC - British-Irish Council

BIIC - British-Irish Intergovernmental Conference

CCRU - Central Community Relations Unit

CIRA - Continuity Irish Republican Army

CLMC - Combined Loyalist Military Command

CRC - Community Relations Council

DUP - Democratic Unionist Party

GFA - Good Friday Agreement

IICD - Independent International Commission on Decommissioning

IFI - International Fund for Ireland

INLA - Irish National Liberation Army

INN - International Negotiation Network

IPLO - Irish People Liberation Army

IRA - Irish Republican Army

IRB - Irish Republican Brotherhood.

LVF - Loyalist Volunteer Force

NIA - Northern Ireland Assembly

NICRA - Northern Ireland Civil Rights Association

NIO - Northern Ireland Office

NIWC - Northern Ireland Women's Coalition

NORAID - North American Aid

NSMC - North-South Ministerial Council

OEA - Organização dos Estados Americanos

ONU - Organização das Nações Unidas

OSCE - Organização para a Segurança e Cooperação na Europa

OUA - Organização da Unidade Africana

PIRA - Provisional Irish Republican Army

PSNI - Police Service in Northern Ireland

PUP - Progressive Unionist Party

RHC - Red Hand Commanders 


$$
\begin{aligned}
& \text { RIRA - Real Irish Republican Army } \\
& \text { RUC - Royal Ulster Constabulary } \\
& \text { SDLP - Social Democratic and Labour Party } \\
& \text { UDA - Ulster Defense Association } \\
& \text { UDP - Ulster Democratic Party } \\
& \text { UE - União Européia } \\
& \text { UFF - Ulster Freedom Fighters } \\
& \text { UKUP - United Kingdom Unionist Party } \\
& \text { UUP - Ulster Unionist Party } \\
& \text { UVF - Ulster Volunteer Force }
\end{aligned}
$$


And the violence causes silence Who are we mistaken? But you see, it's not me It's not my family In your head, in your head They are fighting

With their tanks, and their bombs And their bombs, and their guns

In your head, in your head They are crying (...)

Another mother's breaking Heart is taken over When the violence causes silence We must be mistaken. It's the same old theme Since 1916

In your head, in your head They're still fighting With their tanks, and their bombs And their bombs, and their guns In your head, in your head They are dying

In your head, in your head Zombie, zombie What's in your head, in your head?

(Zombie - The Cranberries) 